\title{
Ergänzende Sozialleistungen im Wohlfahrtsstaat: Das Beispiel Schweden
}

\author{
Gabriella Siögren Lindquist \\ Eskil Wadensjö
}

Umfassende wohlfahrtsstaatliche Systeme, wie sie in den skandinavischen Ländern, aber auch in Deutschland, existieren, fußen nicht einzig auf staatlichen Sozialversicherungssystemen. Daneben gibt es eine Reihe ergänzender Sozialleistungen, die Einkommensausfälle - im Fall von Arbeitslosigkeit, bei vorübergehenden Erwerbsunterbrechungen oder bei Erwerbsunfähigkeit - zusätzlich kompensieren. Der Beitrag stellt das schwedische System ergänzender Sozialleistungen vor und regt vergleichbare Analysen für andere Länder nicht zuletzt als notwendige Grundlage für einen länderübergreifenden Vergleich von Wohlfahrtsstaatssystemen - an. ${ }^{1}$

\section{Einleitung}

Wenn die verschiedenen Wohlfahrtsstaaten und die Auswirkungen ihrer unterschiedlichen Sozialleistungssysteme beschrieben werden, betrachten die meisten Wissenschaftler nur das staatlich organisierte Sozialversicherungssystem. Darüber hinaus kann es aber noch zahlreiche weitere Sozialleistungen geben, die das staatliche Sozialversicherungssystem ergänzen. Dies gilt für die am weitesten entwickelten Wohlfahrtsstaaten, wie beispielsweise die skandinavischen Länder. Wir wollen hier das Beispiel Schweden betrachten, um zu zeigen, wie wichtig dieses „zweite“ System der ergänzenden Sozialleistungen selbst in Staaten ist, die für ihr großzügiges Sozialversicherungssystem bekannt sind.

Die bedeutendsten Ergänzungsleistungen sind solche, die zwischen den Sozialpartnern ausgehandelt werden. Im Folgenden geben wir einen Überblick über derartige, die staatliche Sozialversicherung ergänzenden Sozialleistungen (Abschnitt 2 und 3) und zeigen, wie sie mit dem staatlichen Sozialversicherungssystem verknüpft sind und dieses stärken (Abschnitt 4 und 5). Auch wird die Frage diskutiert, inwieweit ergänzende Sozialleistungen Effekte auf die Einkommensverteilung und das Arbeitskräfteangebot haben (Abschnitt 6). Dass der Staat Einfluss auf die Ausgestaltung ergänzender Sozialleistungen behält, ist das Thema des Abschnitts 7. Das Fazit (Abschnitt 8) plädiert dafür, vergleichbare Zusatzversorgungssysteme anderer Länder genauer zu untersuchen, da erst in der Gesamtschau staatlicher und sie ergänzender Sicherungssysteme die Ausprägungen der jeweiligen Wohlfahrtssysteme erkennbar werden und ländervergleichend betrachtet werden können.

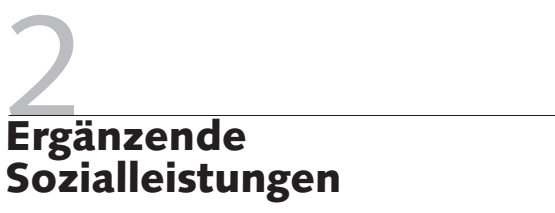

Ausgleichsleistungen - im Folgenden als ergänzende Sozialleistungen gefasst - haben in Schweden eine lange Tradition. Altersruhegeld und Krankengeld, Ausgleichsleistungen bei Arbeitsunfällen und Arbeitslosigkeit gab es bereits vor der Einführung des staatlichen Sozialversicherungssystems. Als Vorgänger der Sozialversicherungssysteme hatten sie Einfluss auf deren Gestaltung. ${ }^{2}$ Auf der anderen Seite wirkten sich die Einführung staatlicher Sozialversicherungssysteme und deren Reformierung wiederum beträchtlich auf die bestehenden ergänzenden Sozialleistungssysteme aus. Außerdem kamen neue Leistungen hinzu, viele davon seit Beginn der 1960er Jahre.

Vereinbarungen über ergänzende Sozialleistungen können auf verschiedenen Ebenen getroffen werden: zwischen Dachverbänden und Tarifgemeinschaften, zwischen nationalen Gewerkschaften und Arbeitgeberverbänden sowie zwischen örtlichen Gewerkschaften und einem einzelnen Arbeitgeber. Von Leistungen, die auf diesem Wege verhandelt werden, profitieren nicht nur die Mitglieder der vertragsabschließenden Gewerkschaften, sondern auch die Nicht-Mitglieder, soweit für sie tarifliche Standards gelten. Nahezu $95 \%$ der Arbeitnehmer im privaten Sektor werden durch derartige tarifliche Vereinbarungen erfasst. Formal betrachtet zahlt der Arbeit- geber die ergänzenden Sozialleistungen. $\mathrm{Da}$ neu verhandelte Sozialleistungen bzw. Änderungen der bestehenden Sozialleistungen aber Teil der Verhandlungsmasse bei Tarifverhandlungen sind, leisten auch die Arbeitnehmer einen Anteil, da sie zugunsten der Sozialleistungen niedrigere Löhne akzeptieren.

Es gibt zwei Grundtypen von ergänzenden Sozialleistungen: Versicherungspläne und Leistungspläne. Bei Versicherungsplänen werden Beiträge an eine Versiche-

\footnotetext{
1 Dieser Aufsatz entstand aufbauend auf Sjögren Lindquist/Wadensjö $(2005,2006)$. Wir danken für die hilfreichen Anmerkungen zu einer früheren Fassung, die anlässlich der Konferenz „Welfare State Change. Conceptualisation, Measurement and Interpretation " (St. Restrup Herregard, Dänemark, 13-15. Januar 2006) entstand. Wir danken auch Silke Bothfeld und Till Müller-Schoell für ihre konstruktiven Kommentare zu diesem Text.

2 Siehe Edebalk et al. (1996) und Rein/Wadensjö (1998) zur allgemeinen Diskussion des Zusammenspiels von Sozialversicherungssystemen und vereinbarungsgebundenen Leistungen.
}

Gabriella Sjörgen Lindquist, $P h D$, ist Wissenschaftlerin am Swedish Institute for Social Research (SOFI), Stockholm University. e-mail: gabriella.sjogren.lindquist@sofi.su.se Eskil Wadensjö, Prof. PhD, ist Professor am Swedish Institute for Social Research (SOFI), Stockholm University, Direktor des Stockholm University Linnaeus Center for Integration Studies (SULCIS) und Research Fellow des Forschungsinstituts zur Zukunft der Arbeit (IZA), Bonn. e-mail: eskil.wadensiö@sofi.su.se

Übersetzung aus dem Englischen: Kristina Vaillant 
rungsgesellschaft abgeführt, die dann Leistungen an diejenige Person erstattet, die den Leistungsfall erfüllt. Bei einem vereinbarten Leistungsplan zahlt der Arbeitgeber die Leistungen direkt an den Empfänger, wenn die in der Vereinbarung vorgesehenen Bedingungen eintreten.

Darüber hinaus gibt es auch eine Bandbreite unterschiedlicher Versicherungsleistungen, die Gewerkschaften ausschließlich für ihre Mitglieder anbieten. Es gibt zwei Hauptarten von Mitgliederversicherungen: Die eine ist verpflichtend, da sie für alle Gewerkschaftsmitglieder oder alle Mitglieder einer Gewerkschaft in einer bestimmten Region gilt. Finanziert wird diese Versicherung entweder durch die Mitgliedsbeiträge oder gesonderte Pflichtzahlungen. Die andere ist eine freiwillige Mitglieder- oder Gruppenversicherung, d.h. hier wird den Mitgliedern einer Gewerkschaft die Option geboten, eine Versicherungspolice abzuschließen.

\section{3 \\ Vereinbarungen in verschiedenen Beschäftigungssektoren}

Am häufigsten werden Tarifvereinbarungen über Leistungen bei bestimmten Formen des Einkommensverlustes in den folgenden vier Bereichen abgeschlossen: Für

- Mitarbeiter der Zentralregierung,

- Mitarbeiter von Kommunal- und Bezirksverwaltungen,

- Angestellte im privaten Sektor,

- Arbeiter im privaten Sektor. ${ }^{3}$

Tabelle 1 zeigt die Anzahl der Beschäftigten in den verschiedenen Sektoren. Diejenigen, die mit unbefristeten Arbeitsverträgen im öffentlichen Sektor (Zentralregierung, Bezirks- und Kommunalverwaltung) beschäftigt sind, profitieren von den in Tarifvereinbarungen abgeschlossenen zusätzlichen Sozialleistungen. Für die Beschäftigten des Privatsektors gilt Vergleichbares nicht unbedingt, da eine Reihe von Unternehmen weder entsprechende Tarifvereinbarungen abgeschlossen hat noch ihren Mitarbeitern vereinbarungsgebundene Versicherungen bietet.

Im Allgemeinen sind die Vereinbarungen an die vier großen Beschäftigungssektoren gebunden, was darauf zurückzu-

\begin{tabular}{|c|c|c|c|c|c|c|}
\hline & \multicolumn{2}{|c|}{ Männer } & \multicolumn{2}{|c|}{ Frauen } & \multicolumn{2}{|c|}{ Gesamt } \\
\hline & Anzahl & $\begin{array}{l}\text { Anteil } \\
\text { in \% }\end{array}$ & Anzahl & $\begin{array}{l}\text { Anteil } \\
\text { in \% }\end{array}$ & Anzahl & $\begin{array}{l}\text { Anteil } \\
\text { in \% }\end{array}$ \\
\hline Zentralregierung & 108.720 & 50 & 106,690 & 50 & 215.410 & 100 \\
\hline Kommunalverwaltung & 160.000 & 21 & 602.620 & 79 & 762.620 & 100 \\
\hline Bezirksverwaltung & 46.580 & 20 & 184,110 & 80 & 230.690 & 100 \\
\hline $\begin{array}{l}\text { Privater Sektor: } \\
\text { Angestellte }\end{array}$ & 726.900 & 57 & 551,900 & 43 & 1.278 .800 & 100 \\
\hline $\begin{array}{l}\text { Privater Sektor: } \\
\text { Arbeiter }\end{array}$ & 798.300 & 70 & 342,800 & 30 & 1.141 .200 & 100 \\
\hline Gesamt & 1.840 .500 & 51 & 1.788 .129 & 49 & 3.628 .720 & 100 \\
\hline
\end{tabular}

führen ist, dass ergänzende Sozialleistungen hauptsächlich zu einer Zeit eingeführt und fixiert worden sind, als Lohnverhandlungen auf nationaler Ebene vorherrschend waren. Heute werden sie im Privatsektor eher branchenbezogen geführt. Das schließt nicht aus, dass es auch heute noch Vereinbarungen über neu eingeführte ergänzende Sozialleistungen gibt, sie sind dann allerdings auch nur für bestimmte Branchen gültig. Ein gutes Beispiel dafür sind die ergänzenden Leistungen während der Elternzeit (vgl. Abschnitt 4.6).

\section{Das staatliche Sozialversicherungssystem und ergänzende Sozialleistungen}

In diesem Abschnitt geben wir einen kurzen Überblick über die Sozialversicherung und das System der ergänzenden Ausgleichsleistungen bei den unterschiedlichen Ursachen des Einkommensverlustes: Arbeitslosigkeit, Berufsunfall, Krankheit, Erwerbsunfähigkeit, Ruhestand und Elternzeit (vgl. dazu ausführlich Sjögren Lindquist/Wadensjö 2005; 2006). Es ist wichtig anzumerken, dass sich die Bestimmungen der Versicherungen auf indexierte Beträge beziehen: den Preisgrundbetrag (price base amount), den erhöhten Preisgrundbetrag (enhanced price base amount) und den Einkommensgrundbetrag (income base amount). ${ }^{4}$

\subsection{ARBEITSLOSENGELD}

Die staatliche Arbeitslosenversicherung zahlt bis zu 300 Tage lang Arbeitslosengeld. Im Jahr 2006 belief sich das Arbeitslosengeld auf $80 \%$ des früheren Einkommens, wobei eine Höchstgrenze festgelegt wurde. Bei der Arbeitslosenversicherung ist diese Grenze niedriger als bei anderen Leistungen der Sozialversicherung (Renten, Elterngeld usw.). Während der ersten hundert Tage der Arbeitslosigkeit liegt der Höchstbetrag bei 20.075 SEK (2.180€) monatlich und wird danach auf 18.700 SEK $(2.030 €)$ monatlich abgesenkt. Die Deckelungen bei anderen Leistungen lagen 2006 bei 24.812 SEK $(2.694 €)$ monatlich.

Im Falle von Beschäftigungsabbau und Arbeitslosigkeit gibt es verschiedene Grundtypen ergänzender Leistungen:

(1) Vereinbarungen über Einkommensgarantien (bis hin zu Arbeitsplatzgarantien) zwischen Gewerkschaften und Arbeitgeberorganisationen;

3 Als Äquivalent für die ergänzenden Sozialleistungen, die abhängig Beschäftigte erhalten, können Selbstständige Versicherungsleistungen von Unternehmen der Versicherungswirtschaft kaufen.

4 Ausgleichszahlungen, Einkommensgrenzen und andere Beträge werden sowohl bei der staatlichen schwedischen Sozialversicherung wie auch bei der betrieblichen Versicherung zumeist auf der Grundlage sogenannter Grundbeträge berechnet. Die Grundbeträge sind an den Preis- oder durchschnittlichen Einkommensindex gekoppelt. Der Grundbetrag wurde als Berechnungsgrundlage erstmals mit der Einführung der Allgemeinen Zusatzrente (ATP) 1960 verwendet. Der Betrag war an den Preisindex der Lebenshaltungskosten gebunden. Mit der jüngsten Rentenreform wurde ein Teil der Rente einkommensabhängig, sodass auch ein Einkommensgrundbetrag eingeführt wurde. Was früher der Grundbetrag war, wird heute als Preisgrundbetrag bezeichnet. In einigen Fällen basieren die Bestimmungen auf einem weiteren Preisgrundbetrag, dem sogenannten erhöhten Preisgrundbetrag. Der Preisgrundbetrag belief sich im Jahr 2006 auf 39.700 Schwedische Kronen (SEK) oder $4.311 €$; der erhöhte Preisgrundbetrag auf 40.500 SEK ( $4.397 €$ ) und der Einkommensgrundbetrag auf 44.500 SEK $(4.831 €)$; berechnet nach dem Wechselkurs vom November 2006: $1 €$ zu 9,21 SEK. 
(2) Leistungen der Mitgliederversicherungen sowie individuelle Versicherungsleistungen über die Gewerkschaften.

Vereinbarungen zu Arbeitsplatz- oder Einkommensgarantien gelten für das gesamte Personal einer Arbeitsstätte, die einen Tarifvertrag abgeschlossen hat; die Mitgliederversicherungen gelten nur für diejenigen, die einer Gewerkschaft mit Kollektivversicherung angehören, und die individuellen Versicherungen für diejenigen Gewerkschaftsmitglieder, die eine individuelle Police abgeschlossen haben.

Ad 1): Die vier Hauptvereinbarungen zu Arbeitsplatz- bzw. Einkommensgarantien gelten

- für Mitarbeiter der Zentralregierung,

- für Mitarbeiter kommunaler Verwaltungen und der Bezirksverwaltungen,

- für Angestellte und Arbeiter im privaten Sektor.

Die Vereinbarung mit der Zentralregierung besagt, dass die Leistung, inklusive der staatlichen Arbeitslosenversicherung, $80 \%$ des vorher bezogenen Gehalts beträgt und genauso lange gezahlt wird wie das staatliche Arbeitslosengeld. Für die Beschäftigten in den Kommunal- und Bezirksverwaltungen gibt es zwei Arten von Leistungen: Erstens eine Abfindungssumme, die sich aus einem Drittel des monatlichen Gehalts multipliziert mit den Jahren der Betriebszugehörigkeit ergibt; und zweitens ein begrenztes Arbeitslosengeld, das während der Periode der Arbeitslosigkeit gezahlt wird. Zusammen mit den staatlichen Leistungen der Sozialversicherung beträgt das Arbeitslosengeld $80 \%$ des früheren Einkommens. Angestellte im privaten Sektor erhalten in den ersten sechs Monaten der Arbeitslosigkeit Leistungen, die sich zusammen mit den Zahlungen der staatlichen Arbeitslosenversicherung auf $70 \%$ des früheren Einkommens bis zu einer Grenze von 20 Preisgrundbeträgen belaufen sowie bis zu $25 \%$ der darüber liegenden Gehaltssumme. Nach diesen sechs Monaten sinkt die Ersatzrate auf 50 \% der Gehaltssumme, die unter 20 Preisgrundbeträgen liegt. Die zeitliche Begrenzung der Zahlungen variiert zwischen sechs und achtzehn Monaten. Arbeiter im privaten Sektor bekommen im Fall der Entlassung eine Abfindungssumme (genannt AGB), die sich - abhängig vom Alter-zwischen 25.000 SEK (2.714€) und 36.000 SEK $(3.909 €)$ bewegt.
Ad 2): Im Jahr 2005 boten acht Gewerkschaften, die dem Dachverband Swedish Confederation of Professional Associations (SACO) angehören, und zwei Gewerkschaften, die Mitglied im Dachverband Federation of White-Collar Workers (TCO) sind, ihren Mitgliedern eine Kollektivversicherung. Hier schwankt der Anspruchszeitraum zwischen 100 und 120 Tagen. In einigen Fällen bieten die Gewerkschaften auch eine individuelle Versicherung für Zusatzleistungen an. Alle SACO-Gewerkschaften sowie eine Reihe der TCO-Mitgliedsgewerkschaften offerieren beispielsweise eine Einkommensversicherung auf individueller Basis. Zusatzversicherungen auf Mitglieder- wie individueller Ebene kompensieren $80 \%$ der Gehaltssumme, die über der Einkommensgrenze der staatlichen Arbeitslosenversicherung liegt. Die Leistungen variieren je nach Vereinbarung. In einigen Fällen wurden Vereinbarungen über Arbeitsplatzgarantien getroffen - womit sich zusätzliche Leistungen von Kollektivversicherungen erübrigen. Für Beschäftigte der Zentralregierung beispielsweise sind Kollektivsicherungen so vorteilhaft, dass sie das Leistungsspektrum individueller Zusatzversicherungen bei Weitem übertreffen.

\subsection{LOHNERSATZLEISTUNGEN BEI ARBEITSUNFÄLLEN}

Alle Erwerbstätigen sind durch die staatlichen Sicherungssysteme bei Arbeitsunfällen versichert. Die Höhe der Lohnersatzleistung entspricht der im Krankheitsfall. Der Leistungsumfang ist sogar besser, weil es bei Leistungen der Berufsunfallversicherung anders als in der Krankenversicherung keine Karenztage gibt. Falls eine dauerhafte Berufsunfähigkeit festgestellt oder für mindestens ein Jahr erwartet wird, erhält der Versicherte eine Versorgungsrente, die das Einkommen bis zu einer Summe von 7,5 Preisgrundbeträgen ersetzt.

Diese staatlichen Leistungen werden für die meisten Beschäftigten durch eine vereinbarungsgebundene Versicherung, als Teil der Tarifvereinbarungen und mit denselben Konditionen für alle Beschäftigungsbereiche, ergänzt. Mitarbeiter, die Verletzungen als Folge von Arbeitsunfällen erleiden, erhalten diese Zusatzleistungen, wenn die Dauer der Berufsunfähigkeit mindestens 14 Tage beträgt. Wird eine dauerhafte Berufsunfähigkeit festgestellt, ersetzt die Versicherung das Einkommen zu $100 \%$. Kein anderes Leistungssystem gewährt höhere Ersatzleistungen. Wegen dieser günstigen Konditionen haben die Gewerkschaften und ihre Mitglieder auf jegliche weitere Leistungen beim Einkommensverlust durch einen Arbeitsunfall verzichtet.

\subsection{LEISTUNGEN IM KRANKHEITSFALL}

Gemäß dem Gesetz zur Lohnfortzahlung im Krankheitsfall sind die Arbeitgeber verpflichtet, ihren Beschäftigten in den ersten zwei Wochen der Erkrankung Krankengeld zu zahlen. Der erste Tag des krankheitsbedingten Ausfalls ist ein Karenztag, vom 2. bis zum 14. Tag erhält der Beschäftigte $80 \%$ des Lohns und anderer Leistungen. Wenn jemand länger als 14 Tage krank geschrieben ist, zahlt die Sozialversicherung ein Krankengeld in Höhe von 80 \% des Gehalts bis zu einer Höchstgrenze von 7,5 Preisgrundbeträgen (im Juli 2006 wurde diese Höchstgrenze auf 10 Preisgrundbeträge angehoben und im Januar 2007 wieder auf die ursprünglichen 7,5 Preisgrundbeträge abgesenkt). Im Jahr 2006 entsprachen 7,5 Preisgrundbeträge einem monatlichen Einkommen von 24.812 SEK (2.694 $€)$. Kommt ein Beschäftigter mit der vereinbarungsgebundenen Versicherung und den Leistungen der staatlichen Sozialversicherung rechnerisch auf mehr als $90 \%$ seiner Bezüge während des krankheitsbedingten Arbeitsausfalls, wird die staatliche Leistung der Sozialversicherung um die entsprechende Summe gekürzt.

Es gibt insgesamt vier ergänzende Vereinbarungen im Bereich des Krankengeldes, von denen die Mehrheit aller Erwerbstätigen profitiert. Sie finden ab dem 15 . Krankheitstag Anwendung. Vom 15. bis zum 90. Krankheitstag erhalten Beschäftigte der Zentralregierung, deren Einkommen die Grenze von 7,5 Preisgrundbeträgen nicht überschreitet, zusätzlich $10 \%$ ihres Gehalts. Diejenigen, deren Einkommen die Summe von 7,5 Preisgrundbeträgen übersteigt, erhalten im gleichen Zeitraum zusätzlich $10 \%$ des Gehalts, das unterhalb der Grenze von 7,5 Preisgrundbeträgen liegt und $90 \%$ der Summe oberhalb der 7,5 Preisgrundbeträge. Vom 91. Tag an erhalten diese Beschäftigten $80 \%$ der über den 7,5 Preisgrundbeträgen liegenden Gehaltssumme.

Die Mitarbeiter der Kommunal- und Bezirksverwaltungen haben vom 15. bis zum 90. Tag ihrer Krankschreibung An- 
spruch auf eine Lohnfortzahlung, die sich zusammen mit dem Krankengeld der staatlichen Sozialversicherung auf $90 \%$ des Gehalts addiert. Zwischen dem 91. und dem 300. Tag der Erkrankung zahlt die Zusatzversicherung $10 \%$ der Gehaltssumme bis zur Grenze von 7,5 Preisgrundbeträgen. Gehaltssummen oberhalb dieser Grenze werden den Beschäftigten der betreffenden Gehaltsgruppen vom 91. Tag an zu $80 \%$ ersetzt.

Angestellte im privaten Sektor erhalten im Krankheitsfall vom 15. bis 90. Tag eine Lohnfortzahlung ihres Arbeitgebers, die sich zusammen mit dem Krankengeld der staatlichen Sozialversicherung auf $90 \%$ des Gehalts addiert. Beschäftigte, deren Gehalt die Höchstgrenze überschreitet, haben Anspruch auf eine zusätzliche Versicherungsleistung vom 90. Tag der krankheitsbedingten Abwesenheit an, und zwar für die Dauer des Leistungsbezuges seitens der staatlichen Sozialversicherung. Ausgeglichen werden in diesem Fall $65 \%$ der Gehaltsanteile, die zwischen der Grenze von 7,5 Preisgrundbeträgen und 20 Einkommensgrundbeträgen liegen sowie 32,5\% der Gehaltssumme ab 20 Einkommensgrundbeträgen bis zu 30 Preisgrundbeträgen. Arbeiter im privaten Sektor haben Anspruch auf eine zusätzliche Ausgleichszahlung vom 15 . bis zum 360 . Tag. Sie beträgt $10 \%$ des Gehalts unterhalb der Höchstgrenze von 7,5 Preisgrundbeträgen. Für Gehaltsanteile oberhalb dieser Grenze gibt es keine Leistungen.

\subsection{ERWERBSUNFÄHIGKEITSRENTE}

Ein Erwerbstätiger, dem eine 25 \%ige Arbeitsunfähigkeit bescheinigt wird, hat seitens des staatlichen Sozialversicherungssystems Anspruch auf eine Erwerbsunfähigkeitsrente. Diese Zahlungen sind einkommensabhängig und betragen $64 \%$ der berechneten Gehaltssumme unterhalb der 7,5 Preisgrundbeträge. Berechnungsgrundlage sind die drei höchsten jemals erhaltenen Jahresgehälter. Erwerbstätige mit niedrigem Einkommen haben Anspruch auf eine gehaltsunabhängige Leistung in Höhe von 2,4 Preisgrundbeträgen, was im Jahr 2006 einer monatlichen Zahlung von 7.940 SEK $(862 €)$ entsprach.

Daneben gibt es vier Vereinbarungen über Zusatzleistungen, die die Mehrheit aller Erwerbstätigen erfassen. Die Zusatzrente im Fall der Erwerbsunfähigkeit beläuft sich für Mitarbeiter der Zentralregierung auf $21 \%$ der Gehaltsumme unterhalb der Grenze von 7,5 Preisgrundbeträgen, auf $81 \%$ der Summe, die zwischen 7,5 und 20 Preisgrundbeträgen liegt, und auf 40,5\% des rentenwirksamen Einkommens, das sich zwischen 20 und 30 Preisgrundbeträgen bewegt. Mitarbeiter von Kommunalund Bezirksverwaltungen, die Anspruch auf eine Erwerbsunfähigkeitsrente haben, erhalten Leistungen abhängig von der Höhe ihrer Gehälter und damit auch abhängig davon, inwieweit diese unterhalb bzw. oberhalb der in der Sozialversicherung festgelegten Grenze liegen. Von dem Gehaltsanteil zwischen 7,5 und 20 Preisgrundbeträgen werden $65 \%$ sowie 32,5\% der Gehaltssumme zwischen 20 und 30 Preisgrundbeträgen ersetzt. Angestellte im privaten Sektor erhalten als Zusatzleistung im Falle der Erwerbsunfähigkeit 15 \% der Gehaltsanteile, die unterhalb der Grenze von 7,5 Preisgrundbeträgen liegen, für die Anteile zwischen 7,5 und 20 Einkommensgrundbeträgen $65 \%$ und für Anteile zwischen 20 und 30 Einkommensgrundbeträgen $32,5 \%$. Beim Anspruch auf eine Erwerbsunfähigkeitsrente durch die staatliche Sozialversicherung können Arbeiter im privaten Sektor eine Zusatzleistung beziehen, deren Höhe vom Einkommen zum Zeitpunkt der Erkrankung abhängig ist.

\subsection{ALTERSRENTE}

Ein neues nationales Rentensystem wurde 1998 eingeführt. Während das alte System dem Leistungsprimat folgte, ist das neue System beitragsbezogen, d.h. zukünftige Altersrenten sind abhängig von den individuellen geleisteten Beiträgen. ${ }^{5}$

Das reformierte Rentenversorgungssystem besteht aus den drei Elementen: Einkommensrente, Prämienrente und Garantierente. Einkommens- und Prämienrente werden auf der Grundlage des Lebenseinkommens berechnet, das bis zur Höhe der Beitragsbemessungsgrenze bis zum Renteneintritt erworben wird. Die Rentenanwartschaften aus jedem einzelnen Jahr entsprechen einem Beitrag von 18,5\% des rentenwirksamen Einkommens des Versicherten; davon sind $16 \%$ für die Einkommensrente bestimmt und 2,5\% für das Prämienrentensystem. Rentenansprüche werden nicht nur auf der Grundlage des Einkommens aus Beschäftigung berechnet, sondern auch selbstständige Tätigkeiten und Leistungen der Sozial- und Arbeitslosenversicherung werden berücksichtigt.
Ferner werden auch Studienzeiten, Militärdienst sowie Erziehungszeiten in die Berechnung der Rentenanwartschaften einbezogen. Die Rente kann frühestens ab dem 61. Lebensjahr bezogen werden. Bei einer niedrigen Einkommens- und Prämienrente wird der Rentenbetrag durch die Garantierente aufgestockt. Die Garantierente wird erst ab dem 65. Lebensjahr ausgezahlt.

Diese Säule der gesetzlichen Rentenversicherung wird ergänzt durch eine zweite Säule berufsbezogener Renten, die für die einzelnen Berufsgruppen unterschiedlich gestaltet ist. Denn diese Zusatzrenten sind zwar staatlich reguliert, die Bedingungen werden allerdings von den Sozialpartnern im Rahmen der Tarifverhandlungen vereinbart. Diese wurden infolge der Reformen in der ersten Säule in jüngster Zeit in beitragsbezogene Systeme umgewandelt.

Die Zusatzrente für die Angestellten im privaten Sektor hatte bislang das zuletzt verdiente Einkommen zur Grundlage. Dabei wurde die Lohnsumme in Teilsegmente unterteilt, die unterschiedlich bewertet werden, sodass niedrige und höhere Einkommen nicht in gleichem Umfang berücksichtigt werden wie mittlere Teile des Einkommens. Für den vollen Rentenanspruch waren 30 Jahre Anwartschaft nötig. Im Jahr 2006 wurde vereinbart, diese Zusatzrente sukzessiv in ein neues, ausschließlich beitragsbezogenes System zu überführen.

Die Zusatzrente der Arbeiter im privaten Sektor ist ebenfalls beitragsbezogen. Der Arbeitgeber zahlt jährlich 3,5 \% des Lohnes als Prämie. In diesem Jahr wurde allerdings die Angleichung an das System der Angestellten im privaten Sektor beschlossen.

Für Beschäftigte der Zentralregierung wurden bereits 2003 die Zusatzrenten in ein beitragsbezogenes System umgewandelt. Allerdings gibt es nach wie vor ein ergänzendes System mit Leistungsprimat für Beschäftigte mit höheren Einkommen. Dieses wird berechnet auf der Basis des Verdienstes der letzten fünf Jahre vor Renteneintritt. Für den vollen Rentenbezug sind 30 Jahre Anwartschaft notwendig.

Für die Beschäftigten der Kommunen existiert auf der Basis einer Vereinbarung von 1989 noch ein System mit Leistungs-

\footnotetext{
5 Im Deutschen wird dieses System daher auch Le
} benseinkommensprinzip genannt. 
primat, bei dem die Einkommenshöhe der letzten sieben Jahre vor Renteneintritt ausschlaggebend ist. Allerdings wurde bereits im Dezember 2005 die Überführung in ein neues System vereinbart mit einer langen Übergangszeit zwischen dem alten und dem neuen System. Ähnlich wie für die Beschäftigten der Zentralregierung wird es bis zu einem bestimmten Einkommen strikt beitragsbezogen sein, während das Einkommen über dem Grenzwert weiterhin dem Leistungsprimat folgt.

\subsection{LEISTUNGEN WÄHREND DER ELTERNZEIT}

Jeder Elternteil hat im Rahmen der Elternzeit Anspruch auf Leistungen für einen Zeitraum von 240 Tagen mit der Option, einen Teil der Tage auf den anderen Elternteil zu übertragen. Insgesamt 60 Tage - die sogenannten Mütter- oder Väter-Monate sind davon jedoch ausgenommen und fest an den jeweiligen Elternteil gebunden.

390 der insgesamt 480 Tage Elternzeit erhalten Eltern $80 \%$ ihres Gehalts bis zur Grenze von 7,5 Preisgrundbeträgen (angehoben auf 10 Preisgrundbeträge im Juli 2006). Der Minimalbetrag für das Elterngeld liegt bei 180 SEK $(20 €)$ pro Tag. Für die übrigen 90 Tage liegt dieser Satz bei $60 \operatorname{SEK}(7 €)$.

Auch für die Elternzeit gibt es verschiedene Zusatzleistungen, die der Arbeitgeber anbietet, die also nicht auf Verhandlungen zwischen den Sozialpartnern zurückgehen. Die Beschäftigten der Zentralregierung erhalten für höchstens 330 Tage pro Elternteil ein zusätzliches Elterngeld, das sich aus $10 \%$ der Gehaltsanteile unter 7,5 Preisgrundbeträgen und $90 \%$ der oberhalb dieser Grenze liegenden Gehaltsanteile zusammensetzt. Die Beschäftigten der Bezirks- und Kommunalverwaltungen erhalten zusätzlich $10 \%$ ihres Monatslohns über einen Zeitraum von ein oder zwei Jahren.
Übersteigt das Gehalt die Grenze von 7,5 Preisgrundbeträgen, hat der Beschäftigte für die Dauer von 270 Tagen zudem Anspruch auf $80 \%$ des darüber liegenden Gehaltsanteils. Für Beschäftige des privaten Sektors wird das Elterngeld durch ein Elterngehalt aufgestockt, das je nach Branche variiert. Arbeiter erhalten als Elterngehalt in der Regel $10 \%$ ihres Lohns für die Dauer von ein bis vier Monaten. Für Angestellte im privatwirtschaftlichen Bereich entspricht das ,typische "Elterngehalt $10 \%$ des Einkommens unter der Preisgrundbetragsgrenze und $90 \%$ der Bezüge oberhalb der Grenze für die Dauer von ein bis vier Monaten.

Im privaten Sektor gibt es andere Varianten: Zusätzliche Leistungen während der Elternzeit, die über einen weit längeren Zeitraum gewährt werden oder die nur von Müttern in Anspruch genommen werden dürfen.

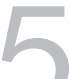 \\ Einkommen oberhalb der staatlichen Sozial- versicherungsgrenze}

Die drei wichtigsten Methoden zur Ergänzung der Sozialversicherungsleistungen haben wir aufgezeigt. Der eine Weg ist, das unter der sozialversicherungsrelevanten Grenze liegende Einkommen aufzustocken. Bei der zweiten Variante werden die Einkommen, die oberhalb der Bemessungsgrenze liegen, berücksichtigt und durch Ersatzzahlungen kompensiert. In der Regel ist die Ersatzrate der zusätzlichen Leistungen für diese Einkommen wesentlich höher als die zusätzlichen Ausgleichsleistungen für die darunter liegenden Beträge. Die dritte Art, Zusatzleistungen zu gewähren, ist, Anspruchszeiträume auszudehnen.
Tabelle 2: Anteil der Beschäftigten mit einem Gehalt über 25.000 SEK $(2.714 €)$ pro Monat im Jahr 2006* - in \%-

\begin{tabular}{|c|c|c|c|}
\hline & Männer & Frauen & Insgesamt \\
\hline Zentralregierung & 59 & 38 & 49 \\
\hline Kommunalverwaltung & 28 & 14 & 17 \\
\hline Bezirksverwaltung & 56 & 29 & 35 \\
\hline Privater Sektor: Angestellte & 69 & 41 & 57 \\
\hline $\begin{array}{l}\text { Privater Sektor: Arbeiter } \\
\text { (Gehalt über } 24.000 \text { SEK }(2.606 €)\end{array}$ & 20 & 9 & 16 \\
\hline \multicolumn{4}{|c|}{$\begin{array}{l}\text { * Die im Sozialversicherungssystem geltende Einkommensgrenze liegt bei 7,5 Preisgrundbeträgen, was einem monatlichen Gehal } \\
\text { von 24.812,50 SEK oder } 2.694 € \text { im Jahr } 2006 \text { entspricht. }\end{array}$} \\
\hline \multicolumn{4}{|c|}{$\begin{array}{l}\text { Quelle: Statistics Swedens Wage Structure Statistics (Schwedens staatliches Statistikamt: } \\
\text { Einkommensstruktur in Schweden). }\end{array}$} \\
\hline
\end{tabular}

Die Kompensationsrate der Zusatzleistungen ist für die Einkommen oberhalb der sozialversicherungsrelevanten Obergrenze am höchsten. Deshalb ist es wichtig zu überprüfen, welche Beschäftigen überhaupt Einkommen über diese Grenze hinaus erzielen. In Tabelle 2 sind die geschätzten Anteile aufgeführt, bezogen auf die für die Altersrente, Erwerbsunfähigkeitsrente, Elternzeit und Krankengeld geltende Einkommensgrenze (7,5 Preisgrundbeträge). Unter den männlichen Beschäftigten der Zentralregierung und der Bezirksverwaltungen sowie unter den Angestellten in der Privatwirtschaft hat deutlich mehr als die Hälfte ein Einkommen oberhalb der Grenze. Auch die weiblichen Beschäftigten erzielen in diesen Beschäftigungsbereichen vielfach ein Einkommen, das oberhalb der sozialversicherungsrelevanten Grenzen liegt - allerdings in weit geringerem Umfang als Männer. Die Beschäftigten der Kommunalverwaltung und Arbeiter bzw. Arbeiterinnen in der Privatwirtschaft erzielen insgesamt deutlich seltener ein Gehalt dieser Höhe, doch immerhin gehören $16 \%$ von ihnen zu dieser Einkommensgruppe. Daher sind Leistungen für oberhalb der Grenze liegende Gehaltsanteile auch für diese Gruppe von Arbeitnehmern von Interesse.

\section{Effekte der Zusatzleistungen}

Zusätzliche Sozialleistungen können vielfältige und unterschiedliche Auswirkungen auf die Beschäftigten, aber auch auf die Wirtschaft als Ganze haben. In diesem Abschnitt werden wir einige dieser Effekte diskutieren.

\subsection{AUSWIRKUNGEN AUF DIE EINKOMMENSVERTEILUNG}

Die verschiedenen Zusatzleistungssysteme sorgen dafür, dass Einkünfte aus Erwerbszeiten auf Zeiten ohne Arbeit übertragen werden. Das bedeutet bzw. setzt voraus, dass Beschäftige auf einen Teil ihres Lohns verzichten. Dieser wird für ergänzende Sozialleistungen angespart, um beispielsweise später eine höhere Rente zu beziehen als die, die das staatliche Sozialversicherungssystem garantiert. Wenn dieses System der Zusatzversicherung so gestaltet ist, dass 
Prämien unabhängig von den individuellen Risiken der Teilnehmer gezahlt werden - und das muss vernünftigerweise der Fall sein -, dann hat das ergänzende Versicherungssystem vergleichbare Umverteilungseffekte wie das staatliche Sozialversicherungssystem. Im Fall individuell zugeschnittener, risikobasierter Versicherungsleistungen würde dieser Verteilungseffekt nicht in gleicher Weise auftreten.

Um Verteilungsfragen geht es auch bei den Zusatzleistungen, die auf Vereinbarungen zwischen den Sozialpartnern basieren, also Bestandteil der Tarifverhandlungen sind, sodass ein Teil des Spielraums für Lohnerhöhungen automatisch für vereinbarungsgebundene Versicherungsleistungen reserviert ist. Der Umfang dieser $\mathrm{Zu}-$ weisung ist bei Gehaltsanteilen oberhalb der Einkommensgrenze zumeist höher als für darunter liegende Einkommen. Es entstünde also ein unvollständiges und teilweise verzerrtes Abbild der Lohnkosten und des Bruttolohns, würde man nicht die Ausgaben der Arbeitgeber für vereinbarungsgemäße Zusatzleistungen - oder alternativ den Wert dieser Vereinbarungen für die Beschäftigten - mit einrechnen.

Die Leistungen des Sozialversicherungssystems haben einen beträchtlichen Umverteilungseffekt bei den Einkommen, und zwar durch die hohe Ersatzrate bei den niedrigen Einkommen im Fall von Arbeitslosigkeit, Krankheit, Erwerbsunfähigkeit, Elternzeit oder Ruhestand. Und das bei Beiträgen, die zumeist nicht versicherungsmathematisch ermittelt wurden. Die ergänzenden Ausgleichsleistungen führen dazu, dass sich die Ersatzraten mehr oder weniger angleichen, und zwar unabhängig vom Einkommen. Das heißt aber nicht unbedingt, dass sie einen Umverteilungseffekt verursachen. Die Zusatzleistungen werden zwar formal gesehen von den Arbeitgebern bezahlt, aber, wie schon angesprochen, auf indirektem Wege von den Arbeitnehmern selbst finanziert, denn sie verzichten zugunsten der ergänzenden Sozialleistungen auf einen Teil ihres Nominallohns; und die verschiedenen Formen der Versicherungsleistungen durch die Gewerkschaften werden durch Beitragsaufkommen oder direkt durch die Versicherten finanziert. Die wichtigsten Schlussfolgerungen hieraus sind, dass die Höhe der Ausgleichszahlungen mehr oder weniger unabhängig vom Einkommen ist, dass die direkt und indirekt geleisteten Beiträge für diejenigen mit höherem Einkommen größer sind und dass es auch in diesem System (öffentliche, betriebliche und mitgliedergebundene Versicherungen zusammengenommen) einen Umverteilungseffekt gibt.

\subsection{AUSWIRKUNGEN AUF ARBEITSKRÄFTEANGEBOT UND ARBEITSZEITEN}

Inwieweit das System ergänzender Sozialleistungen das Angebot an Arbeitskräften und den Umfang der geleisteten Arbeitsstunden beeinflusst, ist eine vielfach diskutierte Frage. Es ist nicht auszuschließen, dass das Angebot zusätzlicher Ausgleichsleistungen einen Anreiz setzt, Arbeit zu suchen und erwerbstätig zu sein. Die zusätzliche Lohnersatzleistung kommt - genauso wie eine Erhöhung des Arbeitsentgelts einer Lohnertragssteigerung gleich. Auf der anderen Seite können diese Zusatzleistungen - nicht anders als die Leistungen des staatlichen Sozialversicherungssystems erwerbstätige Menschen auch dazu motivieren, ihre Arbeit über einen kürzeren oder längeren Zeitraum aufzugeben.

Auch die Gestaltung des Systems der Altersrente kann das Verhalten der Beschäftigten beeinflussen, beispielsweise hinsichtlich der Entscheidung, in welchem Alter Menschen den Arbeitsmarkt verlassen und in Rente gehen. Wir können davon ausgehen, dass ein wesentlicher Einflussfaktor hierfür die Höhe der Rente ist und dass andererseits auch eine Rolle spielt, wie die Rentenhöhe abhängig vom Renteneintrittsalter variiert.

Die Entwicklung des Krankengeldes und auch die von der Krankenversicherung und der Erwerbsunfähigkeitsversicherung geleisteten Ausgleichszahlungen standen in den letzten Jahren im Mittelpunkt der Debatte. Wenn die Leistungen dieser Systeme einen Einfluss auf die Arbeitsaufnahme haben, so kann man logischerweise davon ausgehen, dass sich auch die vereinbarungsgebundenen ergänzenden Leistungen darauf auswirken. Ähnliche Wirkungszusammenhänge werden zwischen Arbeitslosenversicherung und Arbeitslosigkeit vermutet. Insbesondere wird diskutiert, inwieweit der Leistungsumfang der Arbeitslosenversicherung Häufigkeit und Dauer der Arbeitslosigkeit beeinflusst. ${ }^{6}$ Höhere Zahlungen über einen längeren Zeitraum können dazu führen, dass weniger intensiv nach einer Arbeitsstelle gesucht wird und der Arbeitsuchende die Anforderungen an eine Arbeitsstelle hochschraubt. Wenn es diese Effekte gibt, dann muss angenommen werden, dass die Gesamtleistungen und die Gesamtleistungsdauer von entscheidender Bedeutung sind.

\subsection{AUSWIRKUNGEN AUF DIE MOBILITÄT AM ARBEITSMARKT}

Das schwedische Sozialversicherungssystem ist so strukturiert, dass Leistungsansprüche auch bei einem Wechsel des Arbeitsplatzes erhalten bleiben. Im Allgemeinen trifft dies auch auf die vereinbarungsgebundenen Versicherungen und Leistungen zu. Es gibt allerdings Ausnahmen von dieser Regel.

So sehen beispielsweise im privaten Sektor geschlossene Vereinbarungen zum zusätzlichen Elterngeld eine Mindestbeschäftigungszeit im Betrieb von einem Jahr vor. Auch der Umfang der Leistungen ist bei einigen Vereinbarungen von der Dauer der Beschäftigung in dem jeweiligen Unternehmen abhängig, d.h. er steigt mit der Beschäftigungsdauer. Zudem ist es häufig auch Bedingung, dass der Arbeitnehmer nach der Elternzeit für einen festgelegten Zeitraum an seinen Arbeitsplatz zurückkehrt. Diese Regelungen sollen Arbeitnehmer davon abhalten, den Arbeitsplatz während der Elternzeit, oder wenn innerhalb der nächsten Jahre eine Elternzeit geplant wird, zu wechseln. In diesem Regelungszuschnitt drückt sich der Umstand aus, dass entsprechende Vereinbarungen auf Branchenebene - und nicht landesweit - getroffen wurden.

Ähnliche Beispiele sind im Bereich der tariflich vereinbarten betrieblichen Renten zu finden. So gilt hier als Berechnungsbasis nicht das Gesamteinkommen oberhalb der sozialversicherungsrelevanten Grenze, sondern es werden lediglich die Anteile am Gesamteinkommen berücksichtigt, die in einem bestimmten Beschäftigungsbereich verdient wurden. Teilzeitarbeit in einem einzelnen Beschäftigungssektor kombiniert mit Teilzeitarbeit in einem anderen Sektor kann so zu einer wesentlich niedrigeren Rente führen als Vollzeitarbeit innerhalb ein und desselben Beschäftigungssektors, und zwar auch dann, wenn das Gesamtjahreseinkommen in beiden Fällen

\footnotetext{
6 Zu Studien über die Effekte der Arbeitslosenversicherung auf die Arbeitslosigkeit siehe beispielsweise Harkman (1997); Carling et al. (2001) und Røed et al. (2002).
} 
gleich hoch wäre. Dies hält Arbeitnehmer davon ab, zwei Jobs miteinander zu kombinieren, eine Lösung, die in anderer Hinsicht vielleicht durchaus von Vorteil wäre.

\section{Zusatzleistungen im Licht der Wirtschaftspolitik}

Ergänzende Sozialleistungen werden von den Wirtschaftsakteuren selbst und nicht mittels staatlicher Regulierung, also durch Regierung und Parlament (Riksdag), vereinbart. Gleichwohl sind sie dem Einfluss des Staates nicht gänzlich entzogen, da er Zusatzleistungen in unterschiedlicher Weise beeinflussen kann:

(1) Die staatliche Sozialversicherung ist und bleibt der relevante Bezugspunkt für ergänzende Sozialleistungen, d.h. wenn sich das staatliche Sozialversicherungssystem ändert, zieht das in der Regel auch Veränderungen des Systems der Zusatzleistungen nach sich.

(2) Einfluss nimmt der Staat auch durch die Gestaltung des Steuerrechts. Zahlungen für vereinbarungsgebundene Leistungen, Versicherungsbeiträge sowie Leistungen aus Tarifvereinbarungen wurden über die Jahre hinweg steuerrechtlich unterschiedlich behandelt, was jeweils Rückwirkungen auf den Leistungsumfang hat.
(3) Auch kann der Staat Einkommensgrenzen festgelegen, die vorgeben, wie hoch die Ersatzrate der Zusatzleistung sein darf, ohne gleichzeitig die Leistungen der staatlichen Sozialversicherung zu mindern.

(4) Ein vierter Weg eröffnet sich über Allgemeinverbindlicherklärungen, also wenn der Geltungsbereich von Tarifvereinbarungen - auch solcher mit vereinbarungsgebundenen Zusatzleistungen - durch das zuständige Ministerium ausgeweitet wird.

(5) Eine weitere Methode besteht darin, die Einführung von vereinbarungsgebundenen Versicherungen zur Pflicht zu erheben (mit mehr oder weniger detaillierten Vorgaben zu den einzelnen Bestimmungen).

Dass die Einflussnahme des Staates nicht nur graue Theorie ist, zeigt sich an dem Vorhaben der schwedischen Regierung, einen parlamentarischen Ausschuss einzurichten, der sowohl das Sozialversicherungssystem als auch die verschiedenen ergänzenden Systeme prüft.

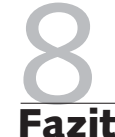

Leistungen zur Kompensation des Einkommensausfalls erfolgen im Wohlfahrtsstaat nicht nur über das staatliche Sozialversicherungssystem. In Schweden, ein
Land, das oftmals als der Archetyp des umfassenden Wohlfahrtsstaates gesehen wird, werden alle Leistungen des staatlichen Sozialversicherungssystems durch zusätzliche Formen der Einkommenskompensation ergänzt. Auch wenn diese zusätzlichen Sozialleistungen vom Umfang her geringer ausfallen als die Leistungen des staatlichen Sozialversicherungssystems, so haben sie dennoch einen hohen Stellenwert. Wir haben die Effekte auf die Einkommensverteilung, das Arbeitskräfteangebot und die Arbeitskräftemobilität diskutiert, die möglicherweise von diesem Zusatzleistungssystem ausgehen. In Grenzbereichen verändern Zusatzleistungen die Anreizstrukturen und beeinflussen so individuelle Entscheidungen, was wiederum Rückwirkungen auf die arbeitsmarkt-, sozial- und gleichstellungspolitische Situation eines Landes haben kann. Ergänzende Sozialleistungen sind - nicht anders als staatliche Sozialversicherungssysteme - Stellschrauben für die Arbeitsmarkt-, Sozial- und Gesellschaftspolitik. Ihr Einfluss ist in anderen Ländern wahrscheinlich mindestens genauso wichtig, wenn nicht noch wichtiger. Daher sind ähnliche Studien zur Situation in anderen Ländern von größtem Interesse. Damit wäre ein Grundstein gelegt, dass Analysen über die Auswirkungen von Einkommensersatzprogrammen künftig bei der Bewertung nationaler Wohlfahrtsstaatssysteme wie auch im Ländervergleich stärker berücksichtigt werden.

\section{LITERATUR}

Carling, K./Holmlund, B./Vejsiu, A. (2001): Do Benefit Cuts Boost Job Findings?, in: Economic Journal, vol. 111, No. 474, S. 766-790 Edebalk, P. G./Ståhlberg, A.-C./Wadensjö, E. (1996): Avtalsrelaterade trygghetssystem vid sjukdom, arbetsskada och förtidspension, in SOU 1996:113, part 2, S. 125-196

Harkman, A. (1997): Arbetslöshetsersättning och arbetslöshetstid - vilken effekt hade sänkningen från 90 till 80 procents ersättningsnivå?, in: Harkman, A./Jansson, F./Källberg, K./Öhrn, L.: Arbetslöshetsersättningen och arbetsmarknadens funktionssätt, Forskningsrapport Ura no 1, National Labour Market Board, Stockholm

Rein, M./Wadensjö, E. (1998): "The relation between social and occupational security", in: Marmor T. R./De Jong, P. R.: Ageing, Social Security and Affordability, Aldershot, S. 28-60
Røed, K./Jensen, P./Thoursie, A. (2002): Unemployment Duration, Incentives and Institutions - A Microeconometric Analysis Based on Scandinavian Countries, Memorandum no 9, 2002, Department of Economics, University of Oslo

Sebardt, G. (2005): Redundancy and the Swedish Model, Uppsala Sjögren Lindquist, G./Wadensjö, E. (2005): "Inte bara socialförsäkringar - kompletterande ersättningar vid inkomstbortfall", Rapport till ESS, no 2 Sjögren Lindquist, G. /Wadensjö, E. (2006): “National Social Insurance not the whole picture. Supplementary compensation in case of loss of income" ${ }^{\prime}$, Report for ESS no 2 